\title{
A Russell-based model for estimating overall and divisional efficiency in two-stage production systems with sets of convex hulls in intermediate products
}

\author{
SAMIRA MALEKI and REZA KAZEMI MATIN*D \\ Department of Mathematics, Karaj Branch, Islamic Azad University, Karaj, Iran \\ e-mail: samira.maleky@gmail.com; rkmatin@kiau.ac.ir
}

MS received 2 May 2018; revised 25 November 2018; accepted 13 December 2018; published online 25 February 2019

\begin{abstract}
Data Envelopment Analysis (DEA) research works have been recently examining production systems with a two-stage network structure. These studies consider system operations that are performed in two stages where intermediate products play dual roles: they are the outputs of the previous stage and inputs to the next stage. This dual role is incompatible with Pareto-Koopmans dominance in activity analysis. Also, disregarding intermediate products in assessing performance of two-stage systems compromises the models. The present work introduces a new production possibility set for two-stage network production systems by considering a convex hull for intermediate products. In addition, new models are introduced for evaluating overall efficiency and divisional efficiency of production units from the Pareto-Koopmans efficiency perspective. The proposed models are developed based on an enhanced Russell graph model for efficiency evaluation of twostage network production structures with convex hulls for intermediate products. Numerical examples are further provided for illustration purposes.
\end{abstract}

Keywords. Data Envelopment Analysis (DEA); two-stage network; intermediate products; convex hull; overall efficiency.

\section{Introduction}

Data Envelopment Analysis (DEA) is a popular method for evaluating relative efficiency of a set of similar DecisionMaking Units (DMUs). DEA methods are especially effective in evaluating efficiency in the presence of multiinput and multi-output variables. As an example, a model introduced by Charnes et al [1] has been extended to apply to different theoretical and practical contexts [2]. DEA has further been utilized in interpreting productivity of complex engineering-economic systems [3-13].

Early DEA methods were utilized to measure efficiency regardless of the internal structure of system operations. However, researchers in the last two decades have paid more attention to system operation in order to investigate inefficiency. The first study on a two-stage network structure using DEA was reported by Charnes et al [14] with an application to army recruitment system. The same twostage network model was then used by many other researchers [15-17].

More recently, several models have been proposed for improving efficiency measurement in two-stage network systems: Wang et al [18] introduced a two-stage method

*For correspondence utilizing Variable Returns to Scale (VRS), where each stage was characterized by independent variables and intermediate products. Rho and An [19] considered slack variables in a model that assessed DMUs with weak efficiency. Kao and Hwang [20] examined the possibility of decomposition in overall system efficiency by considering weights for intermediate products. Liang et al [21] presented a procedure to test the uniqueness of efficiency decomposition. Tone and Tsutsui [22] introduced models that used a production possibility set (PPS) based on a corresponding slack variable.

Chen et al [23] provided a new method for determining efficient projections for inefficient DMUs. They developed an additive efficiency decomposition approach by expressing the overall efficiency as a weighted sum of efficiencies of individual stages. This approach was applicable to both Constant Returns to Scale (CRS) and VRS situations [24]. Chen et al [25, 26] prove that the CRS version of the Chen and Zhu model is equivalent to the output-oriented Kao and Hwang approach [20]. Wang and Chin [27] proposed alternative two-stage network DEA models to show that the overall efficiency of two-stage process could also be formulated as weighted harmonic mean efficiencies of two individual stages.

Liu proposed a two-stage DEA model capable of calculating sub-process and overall process efficiencies 
simultaneously. In a previous work, Kao and Hwang [20] proposed a model where overall efficiency of the whole process had to be calculated prior to computing sub-process efficiency [28]. Du et al [29] proposed a Nash bargaining game model to measure the performance of DMUs with a two-stage structure. Li et al proposed two new two-stage DEA models, including a nonlinear centralized model and a non-cooperative model. Li et al [21] extended the Lian$\mathrm{g}$ et al [21] model by assuming that input to second stage comprised the output from the first stage and additional exogenous input. Yu et al [30] recognized a deficiency in the Wang and Chin [27] model and proposed a novel method for transforming a two-stage process into a onestage process consisting of two independent parallel subprocesses.

Lewis et al [31] used an iterative approach to solve a non-oriented two-stage DEA model using a classical radial objective approach that reduced input quantities and increased output quantities simultaneously. Yu and Shi [32] introduced a two-stage DEA model where intermediate products were freely distributed. Ahmadzadeh et al [33] evaluated the efficiency of a two-stage network structure by utilizing Charnes-Cooper transformation for linearization of fractional program.

Maghbouli et al [34] formulated a two-stage DEA model with undesirable intermediate measures. They proposed cooperative and non-cooperative game theories to assess the relative performance of operational units. Liu et al [35] investigated two-stage DEA models with undesirable input-intermediate output and used free-disposal axiom to construct a PPS. Jianfeng proposed a two-stage DEA model by considering the structure of input and intermediate products measures in efficiency evaluation and decomposition simultaneously [36].

Lim and Zhu [37] formulated equations to obtain frontier projections and divisional efficiency scores through a twostage network DEA. Mahdiloo et al [38] proposed a multiple-criterion two-stage DEA model to find more realistic weights for input and output with better discriminating power than that of traditional two-stage DEA models. Guo et al [39] investigated factors involved in the overall efficiency variations of two-stage additive network DEA approach and examined the relationship of overall efficiency with stage efficiencies and weights. Wanke et al [40] proposed a new combined model based on directional distance functions, which improved the accuracy of efficiency scores under CRS production technologies in two-stage network DEAs.

The main contributions of the referenced models are their respective improvements to measuring efficiency in a two-stage network system. However, they all suffered deficiencies. For instance, Chen et al [41] reported limitations in efficiency measurements related to the different behaviours occurring due to intermediate products. Another researcher, Amir Teimoori [42], considered the presence of perfect and imperfect outputs in a two-stage decision process.

Ignoring intermediate products in assessing the performance of a two-stage network leads to implausible results, thus making it difficult for operators to determine the causes of inefficiencies. Furthermore, some envelopment models used for evaluating overall efficiency are nonlinear programming problems that make efficiency estimation more difficult and less accurate. Therefore, introduction of a method for linearizing models and then exploring a relation between overall (system) efficiency and divisional efficiency of their stages is an important challenge in using nonlinear envelopment models in two-stage production networks.

An important issue being treated in this study is the dissimilar and inconsistent roles of intermediate products in each stage of a two-stage network system. Intermediate products are outputs of a first stage and inputs to a second stage with free disposability assumptions for both stages. This dual-role behaviour imposes a unique structure on production sets in intermediate products. As an example, when being an output, associated production sets are bounded while as an input to the next stage, the same production sets are assumed to be unbounded. This is indicative of an axiomatic foundation of intermediate production sets in network production systems. There is clearly a need for fundamental modifications to the treatment of intermediate products.

It may be claimed that a different behaviour of intermediate products in many two-stage network models is one of the most significant and largely overlooked issues in this art. In addition, no compatibility exists between the two roles described (i.e., two different types of disposability) for a product in a system with the concept of dominance (Pareto-Koopmans efficiency). Thereby, it becomes necessary to revise the model constraints associated with intermediate products in both segments of two-stage network models. This is to comply with the principle of mathematical dominance, which is used to evaluate the system effectiveness.

A bounded production set for intermediate products is applied as per Soltanifar et al [43] using a convexity assumption. As a result, a new set of available intermediate products is introduced, which addresses the afore-mentioned issues.

The purpose of this study is to re-examine the disposability axioms of intermediate products to match management concepts. To this end, the scheme proposed by Ahmadzadeh et al [33] and Soltanifar et al [43] is utilized in envelopment-based network models. Thus, by focusing on the convexity axiom and the principle of mathematical dominance, a new PPS in two-stage systems is introduced that uses a convex hull for intermediate products. A new enhanced Russell graph measure to evaluate overall efficiency of units in a two-stage network structure is then proposed. 
Furthermore, a method for linearizing the proposed model is provided based on Charnes-Cooper transformation. A unique approach for determining divisional efficiency in the new two-stage network set is also produced by formulating a mathematical relation between the efficiency of stages and their corresponding overall efficiency score. A numerical example is presented to demonstrate the proposed models.

The presented work is organized in seven sections. Section 2 briefly reviews related literature on two-stage network DEA. A new approach of using convex hull set for intermediate products is explored in section 3 and a related technology set $\left(T_{C H I}\right)$ is introduced. Section 4 discusses a novel Russell-based model to compute overall efficiency score in the proposed PPS. Section 5 is devoted to introducing a new approach for determining divisional efficiencies in $T_{C H I}$. An illustrative example is presented in section 6 , and section 7 presents the conclusion as well as some suggestions for future research.

\section{Two-stage production systems and intermediate products in DEA literature}

Two different approaches are commonly used in evaluating efficiency of two-stage systems in conventional DEA models.

The first approach estimates efficiency of each division based on the relative efficiency ratio of units. Multiplier-based network DEA models are then derived as per this approach. Estimation of overall and divisional efficiency is generally done using multiplier-based network models. Researchers have presented a wide range of these types of models.

Kao and Hwang [20] calculated efficiency scores of stages one by one under a CRS assumption. Overall efficiency score was then obtained by considering a series relationship between stages as the product of stage efficiencies. It is noted that intermediate product weights are equal in both stages irrespective of the role of intermediate products being an output or an input. Kao and Hwang additionally provided a method for calculating overall efficiency scores of units under VRS [4]. Models were introduced in their studies for calculating divisional efficiency scores and overall efficiency.

The second method utilizes a PPS scheme to measure the efficiency of each division. Later, envelopment models are derived with a two-stage network structure.

Envelopment-based network models are used for determining projections on the efficiency frontier and for calculating overall and divisional efficiencies. Many models have been proposed in this category. Chen et al [24] calculated the overall efficiency in a two-stage system using specific weights in the objective function. Chen et al [25] also introduced a radial version of the envelopment-based network model to compute input-oriented CRS overall efficiency for the units. Chen et al [23] identified the DEA frontier for two-stage processes by imposing redundant constraints for intermediate products in the envelopmentbased network model. Tone and Tsutsui [22] introduced slack-based network DEA models using PPS and explored several models based on intermediate products as fixed or free links.

How intermediate products in a two-stage system are treated and introduced within performance evaluation has a direct impact on results. Wang et al [18] presented separate models for evaluating efficiency of a two-stage network so that intermediate products at each stage are independent of the other stage. Chen and Zhu [26] proposed a two-stage model to maintain dependence between stages. To this end, they treated intermediate products as variables in evaluation so that the variables are identical in both stages. However, their model could not distinguish efficient units from other units. Rho and An [19] suggested a solution to this problem by considering slacks in the constraints of intermediate products.

Kao and Hwang [20] assumed the same weights for intermediate products in both stages for evaluating overall efficiency of a two-stage network. Tone and Tsutsui [22] examined intermediate products in two different fashions by considering the axiomatic foundation of a two-stage network. Tone and Tsutsui [22] referred to these products for evaluating performance as links in the form of fixed and free links. In general, most two-stage network envelopment models consider intermediate products as outputs of a first stage and inputs to the second stage with strong disposability.

Internal operation of the system is the strength in these models; however, models introduced in two-stage networks have considerable deficiencies. Some of these limitations may be due to discrepancy in the variations of utilized concepts of efficiency. Chen et al [41] described some of these limiting inadequacies in detail. As an example, a common limitation of two-stage network DEA models may be the employment of envelopment models for calculating divisional efficiency. It is known that the result of overall efficiency in envelopment models is equal to the efficiency of the first stage. Additionally, multiplier and envelopment models are not necessarily dual models in a two-stage network unlike conventional DEA models. Furthermore, these models demonstrate inadequacies in determining efficient frontier, projection of inefficient DMUs and divisional efficiencies.

Emerged issues could be due to the different nature of particular production sets. It must be noted that a PPS considers intermediate products in two different roles under free disposability assumption. This is incompatible with the concept of mathematical dominance as discussed earlier. Therefore, results of these models cannot directly be used for performance measurements of production units and need to be modified.

The next section introduces a novel approach for imposing a uniform behaviour on intermediate products and for estimating overall and divisional efficiencies. 


\section{Convex hull set for intermediate products in two-stage production systems}

Separate axioms are proposed for each stage in a two-stage network structure in this section. A new PPS is formed for a two-stage network DEA with convex hull set in the intermediate products with employment of these axioms. Relevant properties are then described.

Assume a set of DMUs containing a two-stage internal system where all outputs from the first stage are the only inputs to the second stage. Figure 1 illustrates a two-stage network structure for members of a set of $n$ DMUs [26].

The Kao and Hwang [20] model is applied to explain the main concepts of two-stage DEA models. For each $D M U_{j}$ $(j=1,2, \ldots, n)$ in the first stage, $x_{i j}(i=1,2, \ldots, m)$ inputs are used to produce a set of $D$ intermediate products $z_{d j}(d=$ $1,2, \ldots, D)$. In the second stage, all outputs of the first stage, namely $z_{d j}(d=1,2, \ldots, D)$, are used to produce the final outputs $y_{r j}(r=1,2, \ldots, s)$ where $\boldsymbol{x}=\left(x_{1}, \ldots, x_{m}\right) \in R_{+}^{m}, z=\left(z_{1}, \ldots\right.$, $\left.z_{D}\right) \in R_{+}^{D}$ and $\boldsymbol{y}=\left(y_{1}, \ldots, y_{S}\right) \in R_{+}^{S}$ represent the input vector, intermediate products vector and output vector, respectively. It follows that axioms of "observations inclusion", "convexity" and "strong disposability of inputs" for a set of $D M U$ s in the first stage exist. Thus, the PPS that satisfies these axioms is

$$
\begin{aligned}
T_{1} & =\left\{(\boldsymbol{x}, \boldsymbol{z}): \sum_{j} \lambda_{j} \boldsymbol{x}_{\boldsymbol{j}} \leq \boldsymbol{x}, \sum_{j} \lambda_{j} z_{j}=z, \sum_{j} \lambda_{j}\right. \\
& \left.=1, \lambda_{j} \geq 0 ; j=1, \ldots, n\right\},
\end{aligned}
$$

where variable $\lambda \in R^{n}$ is the vector of intensity variables of the first stage.

Theorem 1 Technology set of the first stage $T_{1}$, defined by Eq. (1), is the minimal set that contains all observations and satisfies the axioms of convexity and strong disposability of inputs.

Proof of Theorem 1 is provided in the appendix.

Axioms of "observations inclusion", "convexity" and "strong disposability of outputs" are postulated for the PPS of the second stage. Hence, the associated production set $T_{2}$ is defined as follows:

$$
\begin{aligned}
T_{2} & =\left\{(z, y): \sum_{j} \mu_{j} z_{j}=z, \sum_{j} \mu_{j} \boldsymbol{y}_{j} \geq \boldsymbol{y}, \sum_{j} \mu_{j}\right. \\
& \left.=1, \mu_{j} \geq 0 ; j=1, \ldots, n\right\},
\end{aligned}
$$

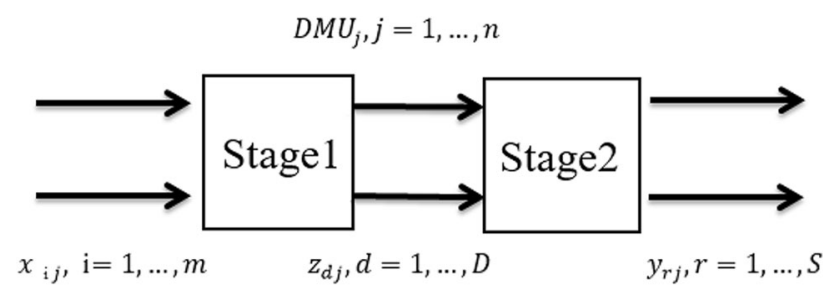

Figure 1. A two-stage production process where each $D M U_{j}$ $(j=1,2, \ldots, n)$ has $m$ inputs, $S$ outputs and $D$ intermediate measures. where the variable $\mu \in R^{n}$ denotes the intensity levels of DMUs for the second stage.

Theorem 2 The second-stage technology set $T_{2}$, defined by (2), is a minimal set that contains all observations and satisfies the axioms of convexity and strong disposability of outputs.

Proof of Theorem 2 is identical to the proof of Theorem 1; hence it is not repeated.

According to proposed axioms for each stage, and under VRS assumption, the overall PPS for a two-stage network with convex hull in intermediate products may be stated as follows:

$$
\begin{aligned}
T_{C H I} & =\left\{(\boldsymbol{x}, \boldsymbol{z}, \boldsymbol{y}): \sum_{j} \lambda_{j} \boldsymbol{x}_{j} \leq \boldsymbol{x}, \sum_{j} \lambda_{j} z_{j}=z, \sum_{j} \mu_{j} z_{j}\right. \\
& =\boldsymbol{z}, \sum_{j} \mu_{j} \boldsymbol{y}_{j} \geq \boldsymbol{y}, \sum_{j} \mu_{j}=1, \sum_{j} \lambda_{j} \\
& \left.=1, \lambda_{j} \geq 0, \mu_{j} \geq 0, j=1, \ldots, n\right\},
\end{aligned}
$$

where $C H I$ represents a set of convex hulls for the intermediate products.

It should be noted that the intermediate products in $T_{C H I}$ are examined by two separate sets of intensity weights $\lambda \in R^{n}$ and $\boldsymbol{\mu} \in R^{n}$. Thus, $\lambda$ determines the relationship between inputs and intermediate products as $\mu$ determines the relation between intermediate products and final outputs. The main difference between the technology expressed in (3) and conventional technology of a two-stage network is that the former allows inequality constraints for the intermediate products while the latter approach suggests equality constraints.

In the described technology, produced output ratio in the first stage is equal to consumed input ratio in the second stage. Therefore, access to resources is restricted and bounded. Thereby, the PPS generated by technology set (3) becomes a subset of the conventional two-stage network PPS.

An illustrative example is presented to compare the overall efficiency and frontier of each stage of a typical two-stage production system using both the proposed and conventional approaches under the conditions of CRS and VRS.

Example 1. Consider a system with four DMUs. Each DMU has one input, one output and one intermediate measure. Table 1 presents the data set.

The overall production technology and PPS for each stage may be clearly seen in figures $2-4$. Bold lines in figure 2 represent the production frontier of stage $1\left(T_{1}\right)$. Note that the efficiency frontier has been expanded by the convex hull of the observations and strong disposability in inputs. Bold lines in figure 3 represent the production frontier of stage $2\left(T_{2}\right)$, where frontier points are obtained with convex hull of the observations and strong disposability axiom in outputs. The thin black lines and dotted lines in figures 2 and 3 represent the conventional 
Table 1. Data set of example 1.

\begin{tabular}{llll}
\hline $\mathrm{dmu}$ & $x$ & $z$ & $y$ \\
\hline$a$ & 1 & 2 & 4 \\
$b$ & 2 & 1 & 1 \\
$c$ & 5 & 4 & 2 \\
$d$ & 2 & 2 & 2 \\
\hline
\end{tabular}

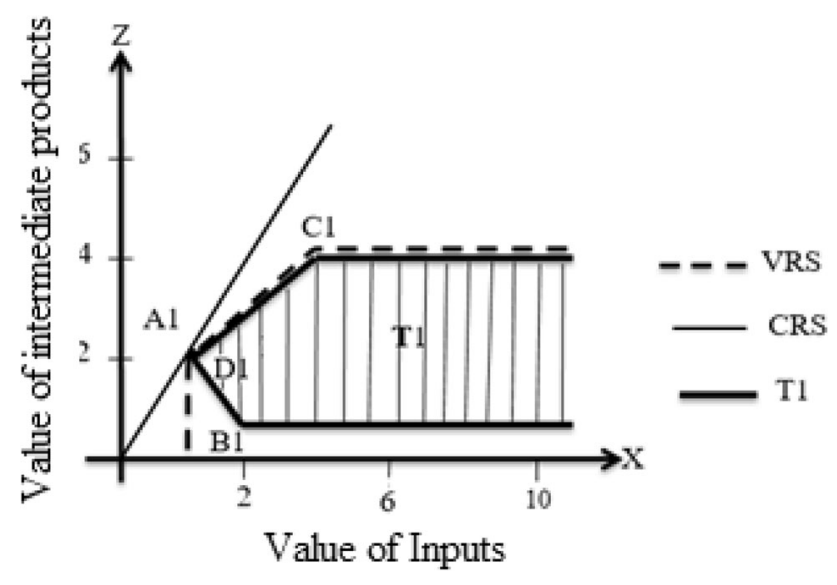

Figure 2. Histogram showing efficient frontier of first stage generated by a two-stage system with the convex hull in intermediate products $\left(T_{1}\right)$. Conventional efficiency frontier is also displayed under the assumptions of CRS and VRS in the first stage.

efficiency frontier, under the assumptions of CRS and VRS, respectively. Clearly, the PPS with the convex hull in the intermediate products is a subset of the PPS under both CRS and VRS assumptions. Note that units $A 1-C 1$ in $T_{1}$ and units $A 2-C 2$ in $T_{2}$ belong to boundary of PPS.

Figure 4 illustrates the 3-D network technology of a convex hull in intermediate products. Note that the

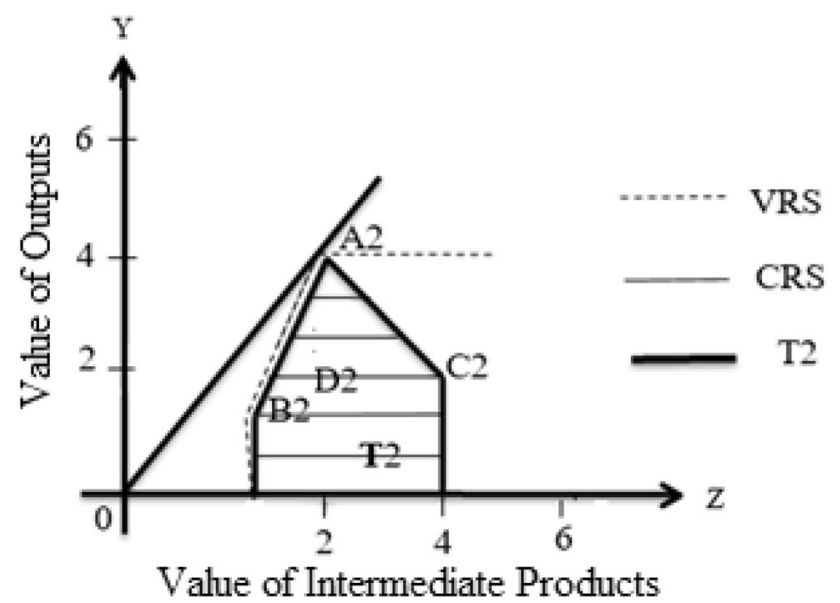

Figure 3. Histogram showing efficient frontier of second stage generated by a two-stage system with convex hull in intermediate products $\left(T_{2}\right)$. Conventional efficiency frontier is also shown under the assumptions of CRS and VRS in the first stage.

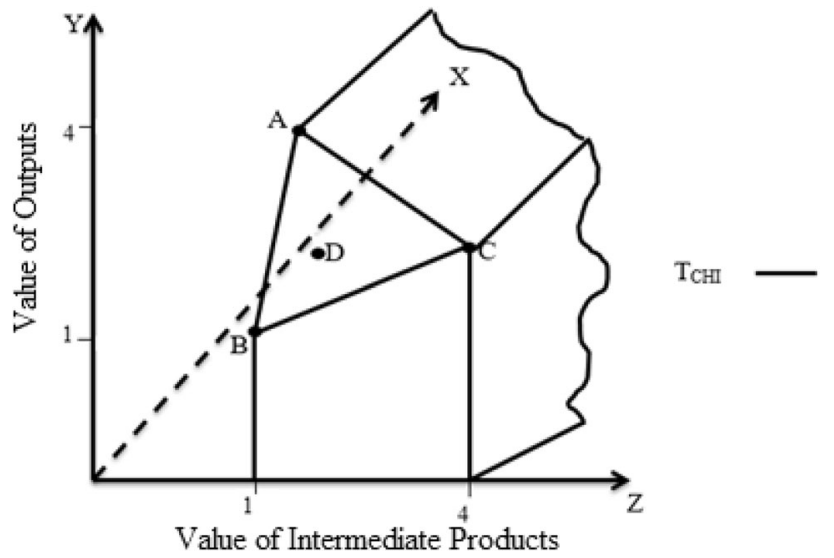

Figure 4. Histogram showing efficient frontier generated by $T_{C H I}$.

intermediate products in $T_{C H I}$ are bounded, and units $A-C$ are located on the boundary of $T_{C H I}$.

Selecting a suitable model for efficiency evaluation would possibly further improve the units on the boundary. More details are presented in the next section.

\section{Determining overall efficiency in $T_{C H I}$}

A new model for evaluating the overall efficiency of production units in $T_{C H I}$ is introduced in this section that utilizes a modified version of the enhanced Russell graph model. A new suggested network model for evaluating overall efficiency of $D M U_{O}$ in $T_{C H I}$ is given as follows:

$$
\begin{aligned}
& E_{o}=\min \left(\hat{w}_{1} E_{o}^{1}+\hat{w}_{2} E_{o}^{2}\right) \\
& =\min \left(\hat{w}_{1} \frac{\frac{1}{m} \sum_{i=1}^{m} \theta_{i}}{\frac{1}{D} \sum_{d=1}^{D} \varphi_{d}}+\hat{w}_{2} \frac{\frac{1}{D} \sum_{d=1}^{D} \bar{\varphi}_{d}}{\frac{1}{s} \sum_{r=1}^{s} \beta_{r}}\right) \\
& \text { s.t. } \quad \sum_{j=1}^{n} \lambda_{j} x_{i j} \leq \theta_{i} x_{i o}, \quad i=1,2, \ldots, m \\
& \sum_{j=1}^{n} \lambda_{j} z_{d j}=\varphi_{d} z_{d o}, \quad d=1,2, \ldots, D \\
& \sum_{j=1}^{n} \mu_{j} z_{d j}=\bar{\varphi}_{d} z_{d o}, \quad d=1,2, \ldots, D \\
& \sum_{j=1}^{n} \mu_{j} y_{r j} \geq \beta_{r} y_{r o}, \quad r=1,2, \ldots, S \\
& \sum_{j=1}^{n} \mu_{j}=\sum_{j=1}^{n} \lambda_{j}=1 \text {, } \\
& \lambda_{j} \geq 0, \mu_{j} \geq 0, \quad j=1,2, \ldots, n \\
& \theta_{i} \leq 1, \quad i=1,2, \ldots, m \\
& \varphi_{d} \geq 1, \quad d=1,2, \ldots, D \\
& \beta_{r} \geq 1, \quad r=1,2, \ldots, S \\
& \bar{\varphi}_{d} \leq 1, \quad d=1,2, \ldots, D
\end{aligned}
$$

where $\theta_{i}, \varphi_{d}, \bar{\varphi}_{d}, \beta_{r}, \lambda_{j}, \mu_{j}$ are decision variables and $\hat{w}_{1}>0$ and $\hat{w}_{2}>0$ are weights such that $\hat{w}_{1}+\hat{w}_{2}=1$, and they may be defined as a constant or a variable; $\theta_{i}$ denotes contraction coefficients of inputs and $\beta_{r}$ represents the expansion coefficients of the final outputs.

Model (4) uses two different patterns for evaluating the overall efficiency in a two-stage system in intermediate 
products: it estimates intermediate products individually according to their roles in each stage. In other words, intermediate products in the first stage have an output role while they have an input role to the second stage. Therefore, in order to evaluate overall efficiency in Model (4), these products are initially scaled with expansion coefficients $\varphi_{d}$ and in the second stage with contraction coefficients $\bar{\varphi}_{d}$. The weights $\hat{w}_{1}$ and $\hat{w}_{2}$ may be determined by employing the model and according to the importance of each division or during optimization. Model (4), which is developed upon Model (3), is an envelopment DEA network model under the assumption of VRS. Since the pattern of Russell's model is compatible with the concept of Pareto-Koopmans efficiency, it is not necessary to consider the slacks in the proposed models. Model (4) is a nonlinear programming model and management priorities may be considered for linearization.

Model (4) can be converted into a linear program (LP) using Charnes-Cooper transformation [33]. In this model, $E_{o}^{1}$ and $E_{o}^{2}$ rely on individual efficiencies of the first and second stages, respectively, to evaluate $D M U_{o}$. Assuming that both stages possess the same importance in evaluation $\left(\hat{w}_{1}=\hat{w}_{2}=\frac{1}{2}\right.$, Model (4) may be rewritten by allowing $t_{1}=$ $\frac{1}{\frac{1}{D} \sum_{d=1}^{D} \varphi_{d}}$ and $t_{2}=\frac{1}{\frac{1}{s} \sum_{r=1}^{s} \beta_{r}}$ and multiplying the constraints by $t_{1}$ and $t_{2}$ separately.

Allowing $\theta_{i}^{1}=t_{1} \theta_{i}, \theta_{i}^{2}=t_{2} \theta_{i}, \quad \varphi_{d}^{1}=t_{1} \varphi_{d}, \quad \varphi_{d}^{2}=t_{2} \varphi_{d}$, $\bar{\varphi}_{d}^{1}=t_{1} \bar{\varphi}_{d}, \quad \bar{\varphi}_{d}^{2}=t_{2} \bar{\varphi}_{d}, \quad \beta_{r}^{1}=t_{1} \beta_{r}, \quad \beta_{r}^{2}=t_{2} \beta_{r}, \quad \lambda_{j}^{1}=t_{1} \lambda_{j}$, $\lambda_{j}^{2}=t_{2} \lambda_{j}, \mu_{j}^{1}=t_{1} \mu_{j}, \mu_{j}^{2}=t_{2} \mu_{j}$, Model (4) transforms into the following LP Model (5):

$$
E_{o}=\min \frac{1}{2}\left(\frac{1}{m} \sum_{i=1}^{m} \theta_{i}^{1}+\frac{1}{D} \sum_{d=1}^{D} \bar{\varphi}_{d}^{2}\right)
$$

Model (5) demonstrates an LP for the two-stage VRS network model, and its optimal solution presents the overall efficiency of the two-stage system. This model evaluates the average efficiencies of the first and second stages $\left(E_{o}=\frac{1}{2}\left(E_{o}^{1}+E_{o}^{2}\right)\right)$. The model together with the constraints related to intermediate products are considered in a convex set of products. It may be observed that Model (5) is always feasible and bounded; i.e., $0<E_{o}^{*} \leq 1$.

Theorem 3 If $\left(\theta_{i}^{1 *}, \theta_{i}^{2 *}, \varphi_{d}^{1 *}, \varphi_{d}^{2 *}, \bar{\varphi}_{d}^{1 *}, \bar{\varphi}_{d}^{2 *}, \beta_{r}^{1 *}, \beta_{r}^{2 *}, \lambda_{j}^{1 *}\right.$, $\left.\lambda_{j}^{2 *}, \mu_{j}^{1 *}, \mu_{j}^{2 *}\right)$ is an optimal solution of Model (5), then

$$
\begin{aligned}
& \left(\theta_{i}^{*}=\frac{\theta_{i}^{1 *}}{t_{1}}=\frac{\theta_{i}^{2 *}}{t_{2}}, \varphi_{d}^{*}=\frac{\varphi_{d}^{1 *}}{t_{1}}=\frac{\varphi_{d}^{2 *}}{t_{2}}, \bar{\varphi}_{d}^{*}=\frac{\bar{\varphi}_{d}^{1 *}}{t_{1}}=\frac{\bar{\varphi}_{d}^{2 *}}{t_{2}},\right. \\
& \left.\beta_{r}^{*}=\frac{\beta_{r}^{1 *}}{t_{1}}=\frac{\beta_{r}^{2 *}}{t_{2}}, \lambda_{j}^{*}=\frac{\lambda_{j}^{1 *}}{t_{1}}=\frac{\lambda_{j}^{2 *}}{t_{2}}, \mu_{j}^{*}=\frac{\mu_{j}^{1 *}}{t_{1}}=\frac{\mu_{j}^{2 *}}{t_{2}}\right)
\end{aligned}
$$

is an optimal solution for Model (4) when $\hat{w}_{1}=\hat{w}_{2}=\frac{1}{2}$. Proof for this theorem is provided in the appendix.

Definition $1 D M U_{o}$ is overall efficient with convex hull in the intermediate products if the optimal value of Model (5) is equal to one; i.e., $E_{o}^{*}=1$.

Example 2. To properly describe Model (5), it is rewritten to evaluate the overall efficiency score for $D M U_{B}$ given in example 1 as follows:

$$
E_{o}^{B}=\min \frac{1}{2}\left(\theta^{1}+\bar{\varphi}^{2}\right)
$$

$$
\begin{aligned}
& \text { s.t. } \quad \sum_{j=1}^{n} \lambda_{j}^{1} x_{i j} \leq \theta_{i}^{1} x_{i o} ; \quad \sum_{j=1}^{n} \lambda_{j}^{2} x_{i j} \leq \theta_{i}^{2} x_{i o}, \quad i=1,2, \ldots, m \\
& \sum_{j=1}^{n} \lambda_{j}^{1} z_{d j}=\varphi_{d}^{1} z_{d o} ; \quad \sum_{j=1}^{n} \lambda_{j}^{2} z_{d j}=\varphi_{d}^{2} z_{d o}, \quad d=1,2, \ldots, D \\
& \sum_{j=1}^{n} \mu_{j}^{1} z_{d j}=\bar{\varphi}_{d}^{1} z_{d o} ; \quad \sum_{j=1}^{n} \mu_{j}^{2} z_{d j}=\bar{\varphi}_{d}^{2} z_{d o}, \quad d=1,2, \ldots, D \\
& \sum_{j=1}^{n} \mu_{j}^{1} y_{r j} \geq \beta_{r}^{1} y_{r o} ; \quad \sum_{j=1}^{n} \mu_{j}^{2} y_{r j} \geq \beta_{r}^{2} y_{r o}, \quad r=1,2, \ldots, S \\
& \sum_{j=1}^{n} \mu_{j}^{1}=t_{1} ; \quad \sum_{j=1}^{n} \mu_{j}^{2}=t_{2} \text {, } \\
& \sum_{j=1}^{n} \lambda_{j}^{1}=t_{1} ; \quad \sum_{j=1}^{n} \lambda_{j}^{2}=t_{2}, \\
& \frac{1}{D} \sum_{d=1}^{D} \varphi_{d}^{1}=1 ; \quad \frac{1}{s} \sum_{r=1}^{s} \beta_{r}^{2}=1 \\
& \theta_{i}^{1} \leq t_{1} ; \quad \theta_{i}^{2} \leq t_{2}, \quad i=1,2, \ldots, m \\
& \varphi_{d}^{1} \geq t_{1} ; \quad \varphi_{d}^{2} \geq t_{2}, \quad d=1,2, \ldots, D \\
& \bar{\varphi}_{d}^{1} \leq t_{1} ; \quad \bar{\varphi}_{d}^{2} \leq t_{2}, \quad d=1,2, \ldots, D \\
& \beta_{r}^{1} \geq t_{1} ; \quad \quad \beta_{r}^{2} \geq t_{2}, \quad r=1,2, \ldots, S \\
& \lambda_{j}^{1} \geq 0 ; \quad \lambda_{j}^{2} \geq 0, \quad j=1,2, \ldots, n \\
& \mu_{j}^{1} \geq 0 ; \quad \mu_{j}^{2} \geq 0, \quad j=1,2, \ldots, n
\end{aligned}
$$




$\begin{array}{lll}\text { s.t. } & \lambda_{1}^{1}+2 \lambda_{2}^{1}+5 \lambda_{3}^{1}+2 \lambda_{4}^{1} \leq 2 \theta^{1} ; & \lambda_{1}^{2}+2 \lambda_{2}^{2}+5 \lambda_{3}^{2}+2 \lambda_{4}^{2} \leq 2 \theta^{2} \\ & 2 \lambda_{1}^{1}+\lambda_{2}^{1}+4 \lambda_{3}^{1}+2 \lambda_{4}^{1}=1 ; & 2 \lambda_{1}^{2}+\lambda_{2}^{2}+4 \lambda_{3}^{2}+2 \lambda_{4}^{2}=\varphi^{2} \\ & 2 \mu_{1}^{1}+\mu_{2}^{1}+4 \mu_{3}^{1}+2 \mu_{4}^{1}=\bar{\varphi}^{1} ; & 2 \mu_{1}^{2}+\mu_{2}^{2}+4 \mu_{3}^{2}+2 \mu_{4}^{2}=\bar{\varphi}^{2} \\ & 4 \mu_{1}^{1}+\mu_{2}^{1}+2 \mu_{3}^{1}+2 \mu_{4}^{1} \geq \beta^{1} ; & 4 \mu_{1}^{2}+\mu_{2}^{2}+2 \mu_{3}^{2}+2 \mu_{4}^{2} \geq 1 \\ & \lambda_{1}^{1}+\lambda_{2}^{1}+\lambda_{3}^{1}+\lambda_{4}^{1}=t_{1} ; & \lambda_{1}^{2}+\lambda_{2}^{2}+\lambda_{3}^{2}+\lambda_{4}^{2}=t_{2} \\ & \mu_{1}^{1}+\mu_{2}^{1}+\mu_{3}^{1}+\mu_{4}^{1}=t_{1} & \mu_{1}^{2}+\mu_{2}^{2}+\mu_{3}^{2}+\mu_{4}^{2}=t_{2} \\ & \theta^{1} \leq t_{1}, \theta^{2} \leq t_{2} & \bar{\varphi}^{1} \leq t_{1}, \bar{\varphi}^{2} \leq t_{2} \\ & \beta^{1} \geq t_{1}, \quad 1 \geq t_{2} & \varphi^{2} \geq t_{2}, 1 \geq t_{1} \\ & \lambda_{j}^{1} \geq 0, \lambda_{j}^{2} \geq 0, \mu_{j}^{1} \geq 0, \mu_{j}^{2} \geq 0, & j=1, \ldots, 4 .\end{array}$

On solving Model (6), optimal overall efficiency score for $\mathrm{DMU}_{\mathrm{B}}$ is obtained as $E_{o}^{B *}=0.625$. Similarly, the proposed model can evaluate the overall efficiency scores for other DMUs in example 1. Table 2 presents the results of this calculation.

Thus, $D M U_{A}$ with convex hull in the intermediate products is overall efficient while other DMUs are inefficientall. Optimally, scaling coefficients show a value of 1 in evaluation of $D M U_{A}$ under Model (5). Therefore, $D M U_{A}$ is a member of the referenced set and may be introduced as a target for frontier projection of inefficient DMUs.

Regarding evaluation of $D M U_{B}$ under Model (5), optimal values are obtained as $t_{1}^{*}=0.5, t_{2}^{*}=1, \theta^{1 *}=0.25, \bar{\varphi}^{2 *}=$ $1, \varphi^{1 *}=1$ and $\beta^{2 *}=1$. Using the defined formulas, efficiencies of each stage and targets are calculated. Results indicate that efficiency of the first stage is equal to $E_{B}^{1 *}=$ $\theta^{1 *}=0.25 \quad$ and $\quad \theta_{B}^{*}=\frac{\theta^{1 *}}{t_{1}^{*}}=\frac{0.25}{0.5}=\frac{1}{2}, \varphi_{B}^{*}=\frac{\varphi^{1 *}}{t_{1}^{*}}=\frac{1}{0.5}=2$. This shows that the optimal solution for Model (5) is equal to the optimal solution of Model (4) $\left(E_{B}^{1 *}=\frac{1}{2}=0.25\right)$, and the obtained projection from results of the first stage from Model (4) could be determined as $\left(\theta_{B}^{*} x_{B}=x_{B}^{*}, \varphi_{B}^{*} z_{B}=z_{B}^{*}\right)$, i.e., $x_{B}^{*}=1$ and $z_{B}^{*}=2$.

Thus, despite the fact that $D M U_{B}$ is located on the boundary, it may be targeted on $D M U_{A}$ due to the possibility of a change in the convex hull of the intermediate products. Therefore, $D M U_{B}$ is not efficient in the first stage.

Additionally, the second stage scaling factors for Model (4) are $\bar{\varphi}_{B}^{*}=\frac{\bar{\varphi}^{2 *}}{t_{2}^{*}}=1$ and $\beta_{B}^{*}=\frac{\beta^{2 *}}{t_{2}^{*}}=1$ based on results. Thus, $D M U_{B}$ is efficient in the second stage $\left(E_{B}^{2 *}=\bar{\varphi}^{2 *}=1\right)$ and is located on the efficient frontier.

Table 2. The overall efficiency scores for four DMUs in example 1.

\begin{tabular}{lcccc}
\hline$d m u$ & $A$ & $B$ & $C$ & $D$ \\
\hline$\theta_{o}^{*}$ & 1 & 0.625 & 0.625 & 0.5 \\
\hline
\end{tabular}

On evaluating $D M U_{D}$ under Model (5), optimal values are obtained as $t_{1}^{*}=1, t_{2}^{*}=0.5, \theta^{1 *}=0.5, \bar{\varphi}^{2 *}=$ $0.5, \varphi^{1 *}=1$ and $\beta^{2 *}=1$. Efficiency of the first and second stages are equal to $E_{D}^{1 *}=\theta^{1 *}=0.5$ and $E_{D}^{2 *}=\bar{\varphi}^{2 *}=0.5$, respectively. Therefore, $D M U_{D}$ is inefficient in both stages. Obtained results for scale factors in the first stage of Model (4) using these results are equal to $\theta_{D}^{*}=\frac{\theta^{1 *}}{t_{1}^{*}}=\frac{0.5}{1}=0.5$ and $\varphi_{D}^{*}=\frac{\varphi^{1 *}}{t_{1}^{*}}=\frac{1}{1}$, and in the second stage, $\bar{\varphi}_{D}^{*}=\frac{\bar{\varphi}^{2 *}}{t_{2}^{*}}=1$ and $\beta_{D}^{*}=\frac{\beta^{2 *}}{t_{2}^{*}}=\frac{1}{0.5}=2$; thus $E_{D}^{1 *}=E_{D}^{2 *}=0.5$. Furthermore, input and output of $D M U_{D}$ can be projected on the input and output of $D M U_{A}$ using Model (4).

It must be noted that Model (5) focuses on sets of convex hulls in intermediate products to evaluate performance. Thus, these products may be changed only in this bounded hull. Model (5) scales the intermediate products in the first stage with expansion coefficients, and in the second stage with coefficients of contraction.

Therefore, while Model (5) preserves intermediate products at the same levels in both stages, overall efficiency is evaluated so that the level of production of intermediate products in the first stage is higher than the rate of product consumption in the second stage. These performance evaluations are consistent with production conditions.

\section{Determining divisional efficiency in $T_{C H I}$}

A new network DEA model that determines divisional efficiency for DMUs is introduced in this section. For this purpose, production sets (1) and (2) are used in calculating divisional efficiency.

Efficiency scores for $D M U_{o}$ in the first and second stages are estimated by the following models:

$$
E_{o}^{1}=\min \frac{\frac{1}{m} \sum_{i=1}^{m} \theta_{i}}{\frac{1}{D} \sum_{d=1}^{D} \varphi_{d}}
$$




$$
\begin{aligned}
& \begin{array}{ll}
\text { s.t. } & \sum_{j=1}^{n} \lambda_{j} x_{i j} \leq \theta_{i} x_{i o}, \quad i=1, \ldots, m \\
& \sum_{j=1}^{n} \lambda_{j} z_{d j}=\varphi_{d} z_{d o}, \quad d=1, \ldots, D
\end{array} \\
& \sum_{j=1}^{n} \lambda_{j}=1 \\
& \lambda_{j} \geq 0, \quad j=1, \ldots, n \\
& \theta_{i} \leq 1, \quad i=1, \ldots, m \\
& \varphi_{d} \geq 1, \quad d=1, \ldots, D \\
& E_{o}^{2}=\min \frac{\frac{1}{D} \sum_{d=1}^{D} \bar{\varphi}_{d}}{\frac{1}{s} \sum_{r=1}^{s} \beta_{r}} \\
& \text { s.t. } \quad \sum_{j=1}^{n} \mu_{j} z_{d j}=\bar{\varphi}_{d} z_{d o}, \quad d=1, \ldots, D \\
& \sum_{j=1}^{n} \mu_{j} y_{r j} \geq \beta_{r} y_{r o}, \quad r=1, \ldots, s \\
& \sum_{j=1}^{n} \mu_{j}=1 \text {, } \\
& \mu_{j} \geq 0, \quad j=1, \ldots, n \\
& \beta_{r} \geq 1, \quad r=1, \ldots, s \\
& \bar{\varphi}_{d} \leq 1, \quad d=1, \ldots, D .
\end{aligned}
$$

Models (7) and (8) may be viewed as an enhanced Russell graph by assuming VRS. $E_{o}^{1}$ and $E_{o}^{2}$ represent efficiencies of the first and second stages, respectively, in these models. These fractional models are transformed to LPs by applying the Charnes-Cooper transformation to produce Models (9) and (10):

$$
E_{o}^{1}=\min \frac{1}{m} \sum_{i=1}^{m} \theta_{i}
$$

$\begin{array}{lll}\text { s.t. } & \sum_{j=1}^{n} \lambda_{j} x_{i j} \leq \theta_{i} x_{i o}, & i=1, \ldots, m \\ & \sum_{j=1}^{n} \lambda_{j} z_{d j}=\varphi_{d} z_{d o}, & d=1, \ldots, D \\ & \sum_{j=1}^{n} \lambda_{j}=t & \\ & \frac{1}{D} \sum_{d=1}^{D} \varphi_{d}=1, & \\ \lambda_{j} \geq 0, & j=1, \ldots, m \\ \theta_{i} \leq t, & i=1, \ldots, m \\ \varphi_{d} \geq t, & d=1, \ldots, D\end{array}$

$$
E_{o}^{2}=\min \frac{1}{D} \sum_{d=1}^{D} \bar{\varphi}_{d}
$$

$$
\begin{array}{lll}
\text { s.t. } & \sum_{j=1}^{n} \mu_{j} z_{d j}=\bar{\varphi}_{d} z_{d o}, & d=1, \ldots, D \\
& \sum_{j=1}^{n} \mu_{j} y_{r j} \leq \beta_{r} y_{r o}, & r=1, \ldots, s \\
& \sum_{j=1}^{n} \mu_{j}=t, & \\
& \frac{1}{s} \sum_{r=1}^{s} \beta_{r}=1, & \\
\mu_{j} \geq 0, & j=1, \ldots, n \\
\beta_{r} \geq t, & r=1, \ldots, s \\
\bar{\varphi}_{d} \leq t, & d=1, \ldots, D
\end{array}
$$

Interpretation of the Models (9) and (10) is similar to the interpretation of enhanced Russell graph by assuming VRS. The only difference would be the use of equality restriction in output constraints of the first stage of Model (9) and in the input constraints of Model (10). Note that the overall efficiency score is the arithmetic mean of efficiency scores of all stages.

Definition 2 First (or second) division of $D M U_{o}$ is efficient with respect to the set of convex hull in the intermediate products, if and only if the optimal value of Model
Table 3. Stage efficiency scores of four units in the first stage for example 1 .

\begin{tabular}{lcc}
\hline DMU & Stage 1 & Stage 2 \\
\hline$A$ & 1 & 1 \\
$B$ & 0.25 & 1 \\
$C$ & 1 & 0.25 \\
$D$ & 0.5 & 0.5 \\
\hline
\end{tabular}

(9) or Model (10) is equal to one; i.e., $E_{o}^{1 *}=1$ (or $E_{o}^{2 *}=1$ ).

To examine divisional efficiency scores in example 1, Models (9) and (10) are applied and results are reported in table 3 . It is observed that results with the interpretations presented for DMUs in the previous section are the same. Results indicate that $D M U_{\mathrm{A}}$ is efficient with convex hull in intermediate products at both stages and the remaining DMUs are inefficient at least at one stage. As observed, overall efficiency score is the average of the first and the second stage efficiency scores.

\section{An illustrative example}

This section concerns application of the produced models to assess overall and divisional efficiency scores for the data set used in Kao and Hwang [20]. Performance of 24 nonlife insurance companies in Taiwan was assessed in that study using a two-stage system in series.

Kao and Hwang [20] processed this example in two stages: premium acquisition and profit generation. The first stage uses two inputs to produce two intermediate products, which are consumed by the second stage to produce two final outputs. Inputs are considered to be employee operational and insurance expenses; intermediate products are directly written and reinsurance premiums, and the final two outputs are the underwriting and investment profits. Table 4 presents the data set of this example in the Kao and Hwang study [20].

Table 5 lists the calculated overall and divisional efficiency scores of the DMUs using Models (5), (9) and (10). Note that in the newly proposed method for evaluation of non-life insurance companies, intermediate products (directly written and reinsurance premiums) are allowed to vary only in the convex hull of the observed data. Products are boundaries; therefore, these products must exist in an acceptable range for performance evaluation of the companies.

As observed, overall efficiency and divisional efficiency score values for both stages of $D M U_{5}$ and $D M U_{22}$ are equal to one. Therefore, these DMUs are generally efficient in the new production set and lie on the VRS frontier when evaluated by the proposed network model. DMUs for 1, 2, $9,12,15,18,19$ and 24 in the first division are efficient with convex hull in the intermediate products. DMUs for 3, 17 and 20 in the second division are efficient with respect to 
Table 4. Data set of the example.

\begin{tabular}{|c|c|c|c|c|c|c|}
\hline DMU & $\begin{array}{l}\text { Operational } \\
\text { expenses } \boldsymbol{x}_{\boldsymbol{1}}\end{array}$ & $\begin{array}{c}\text { Insurance } \\
\text { expenses } \boldsymbol{x}_{2}\end{array}$ & $\begin{array}{l}\text { Direct written } \\
\text { premiums } z_{1}\end{array}$ & $\begin{array}{l}\text { Reinsurance } \\
\text { premiums } z_{2}\end{array}$ & $\begin{array}{l}\text { Underwriting } \\
\text { profit } \boldsymbol{y}_{\boldsymbol{1}}\end{array}$ & $\begin{array}{c}\text { Investment } \\
\text { profit } \boldsymbol{y}_{\boldsymbol{2}}\end{array}$ \\
\hline 1 & $1,178,744$ & 673,512 & $7,451,757$ & 856,735 & 984,143 & 681,687 \\
\hline 2 & $1,381,822$ & $1,352,755$ & $10,020,274$ & $1,812,894$ & $1,228,502$ & 834,754 \\
\hline 3 & $1,177,494$ & 592,790 & $4,776,548$ & 560,244 & 293,613 & 658,428 \\
\hline 4 & 601,320 & 594,259 & $3,174,851$ & 371,863 & 248,709 & 177,331 \\
\hline 5 & $6,699,063$ & $3,531,614$ & $37,392,862$ & $1,753,794$ & $7,851,229$ & $3,925,272$ \\
\hline 6 & $2,627,707$ & 668,363 & $9,747,908$ & 952,326 & $1,713,598$ & 415,058 \\
\hline 7 & $1,942,833$ & $1,443,100$ & $10,685,457$ & 643,412 & $2,239,593$ & 439,039 \\
\hline 8 & $3,789,001$ & $1,873,530$ & $17,267,266$ & $1,134,600$ & $3,899,530$ & 622,868 \\
\hline 9 & $1,567,746$ & 950,432 & $11,473,162$ & 546,337 & $1,043,778$ & 264,098 \\
\hline 10 & $1,303,249$ & $1,298,470$ & $8,210,389$ & 504,528 & $1,697,941$ & 554,806 \\
\hline 11 & $1,962,448$ & 672,414 & $7,222,378$ & 643,178 & $1,486,014$ & 18,259 \\
\hline 12 & $2,592,790$ & 650,952 & $9,434,406$ & $1,118,489$ & $1,574,191$ & 909,295 \\
\hline 13 & $2,609,941$ & $1,368,802$ & $13,921,464$ & 811,343 & $3,609,236$ & 223,047 \\
\hline 14 & $1,396,002$ & 988,888 & $7,396,396$ & 465,509 & $1,401,200$ & 332,283 \\
\hline 15 & $2,184,944$ & 651,063 & $10,422,297$ & 749,893 & $3,355,197$ & 555,482 \\
\hline 16 & $1,211,716$ & 415,071 & $5,606,013$ & 402,881 & 854,054 & 197,947 \\
\hline 17 & $1,453,797$ & $1,085,019$ & $7,695,461$ & 342,489 & $3,144,484$ & 371,984 \\
\hline 18 & 757,515 & 547,997 & $3,631,484$ & 995,620 & 692,731 & 163,927 \\
\hline 19 & 159,422 & 182,338 & $1,141,951$ & 483,291 & 519,121 & 46,857 \\
\hline 20 & 145,442 & 53,518 & 316,829 & 131,920 & 355,624 & 26,537 \\
\hline 21 & 84,171 & 26,224 & 225,888 & 40,542 & 51,950 & 6491 \\
\hline 22 & 15,993 & 10502 & 52,063 & 14,574 & 82,141 & 4181 \\
\hline 23 & 54,693 & 28,408 & 245,910 & 49,864 & 0.1 & 18,980 \\
\hline 24 & 163,297 & 235,094 & 476,419 & 644,816 & 142,370 & 16,976 \\
\hline
\end{tabular}

Table 5. The overall and divisional efficiency scores.

\begin{tabular}{clll}
\hline DMU & $\begin{array}{c}\text { Overall } \\
\boldsymbol{E}_{\boldsymbol{o}}^{*}\end{array}$ & $\begin{array}{c}\text { Stage } 1 \\
\boldsymbol{E}_{\boldsymbol{o}}^{1 *}\end{array}$ & $\begin{array}{c}\text { Stage } 2 \\
\boldsymbol{E}_{\boldsymbol{o}}^{2 *}\end{array}$ \\
\hline 1 & 0.73607 & 1 & 0.47214 \\
2 & 0.68733 & 1 & 0.37467 \\
3 & 0.80172 & 0.60345 & 1 \\
4 & 0.35465 & 0.47730 & 0.23199 \\
5 & 1 & 1 & 1 \\
6 & 0.66640 & 0.92913 & 0.40367 \\
7 & 0.48009 & 0.45914 & 0.50104 \\
8 & 0.57433 & 0.69686 & 0.45179 \\
9 & 0.64469 & 1 & 0.28939 \\
10 & 0.53983 & 0.42404 & 0.65562 \\
11 & 0.35321 & 0.67016 & 0.036259 \\
12 & 0.78385 & 1 & 0.56770 \\
13 & 0.49211 & 0.71566 & 0.26856 \\
14 & 0.47144 & 0.43575 & 0.50712 \\
15 & 0.84427 & 1 & 0.68855 \\
16 & 0.56785 & 0.75571 & 0.37998 \\
17 & 0.65946 & 0.31893 & 1 \\
18 & 0.66768 & 1 & 0.33537 \\
19 & 0.69655 & 1 & 0.39310 \\
20 & 0.83523 & 0.67046 & 1 \\
21 & 0.50402 & 0.74107 & 0.26697 \\
22 & 1 & 1 & 1 \\
23 & 0.48323 & 0.96647 & 0.10377 \\
24 & 0.60351 & 1 & 0.20703 \\
\hline & & & \\
\hline
\end{tabular}

the convex hull in the intermediate products. Other units are inefficient in both divisions.

Overall efficiency scores are calculated by averaging the efficiency of all stages, i.e., $\left(\left(E_{o}^{*}=\frac{1}{2}\left(E_{o}^{1 *}+E_{o}^{2 *}\right)\right)\right.$.

Analysing the results in this example, it is observed that the proposed models in this work are effective in assessing performance of DMUs.

Additionally, the new models are capable of identifying the sources of inefficiencies in a two-stage network and help managers in addressing them. For example, results obtained from Models (5), (9) and (10) for $D M U_{1}$ are, respectively, $E_{1}^{*}=0.73607, E_{1}^{1 *}=1$ and $E_{1}^{2 *}=0.47214$. Therefore, inefficiency of $D M U_{1}$ is due to poor performance in the second stage. Obtaining scaling coefficients from Model (10) enables a manager to take necessary actions to correct inefficiencies. Therefore, the newly introduced production set and the associated performance evaluation method presented in this paper are valuable and have practical utility in terms of production evaluation and performance management.

\section{Conclusion}

Two-stage network models are important in DEA due to their utility in complex models. Conventional DEA models with a two-stage network structure treat 
intermediate products in a dual fashion as input or output. This duality in roles can lead to inconsistencies in efficiency evaluation and Pareto-Koopmans dominance concepts.

This work investigates imposition of a uniform behaviour on intermediate products with an axiomatic approach. Further, a new PPS in two-stage production systems is proposed by focusing on dominance notion and convexity axiom using a set of convex hulls for intermediate products under the assumption of VRS.

An enhanced Russell graph measure is proposed to assess overall efficiency scores based on a new PPS. A method for converting the new non-linear model to a linear programming equivalent is next introduced using Charnes-Cooper transformation. The proposed enhanced Russell graph models also provide assessment of divisional efficiency under the assumption of VRS. In addition, it is proposed to use an average of stage efficiencies to represent overall efficiency of units. Finally, illustrative examples are presented to demonstrate and examine the new approach.

A significant advantage of the proposed models is their capability in utilization of input, output and intermediate products as complete vectors in evaluation of respective units. Therefore, as results indicate, the proposed models are consistent with the concept of Pareto-Koopmans efficiency because they consider sets of convex hulls for intermediate products in performance assessment of twostage network units. Additionally, using the same proposed models, it may be possible to determine sources of inefficiency for specific production units. This may prove valuable and beneficial from an organizational management perspective.

\section{Appendix}

(a) Theorem 1. The first-stage technology or $T_{1}$, which is defined in set (4), is the minimal set that contains all observations and satisfies the axioms of strong disposability of inputs and convexity.

Proof Assume that technology T satisfies the axioms (A1)(A3). It is shown that $T_{1} \subseteq T$.

Let $\left(\left(x_{1}, z_{1}\right) \in T_{1}\right)$ so there exist non-negative intensity weights $\lambda_{1}, \ldots, \lambda_{n}$ for which $\sum_{j} \lambda_{j}=1$ and $\left\{\begin{array}{l}x_{1} \geq \sum_{j} \lambda_{j} x_{j} \\ z_{1}=\sum_{j} \lambda_{j} z_{j}\end{array}\right.$. Since $\mathrm{T}$ satisfies (A1), it includes any observed $\left(\boldsymbol{x}_{j}, \boldsymbol{z}_{j}\right)$, $j=1, \ldots, N$ and from (A2), therefore, $\sum_{j} \lambda_{j}\left(x_{j}, z_{j}\right)=\left(\sum_{j} \lambda_{j} x_{j}, \sum_{j} \lambda_{j} z_{j}\right) \in T . \mathrm{T}$ clearly satisfies (A3) in input side and that in turn indicates that $\left(x_{1}, z_{1}\right) \in T$.

Proof is complete. Note that proof for Theorem 2 is identical to proof for Theorem 1 . (b) Theorem 3. If $\left(\theta_{i}^{1 *}, \theta_{i}^{2 *}, \varphi_{d}^{1 *}, \varphi_{d}^{2 *}, \bar{\varphi}_{d}^{1 *}, \bar{\varphi}_{d}^{2 *}, \beta_{r}^{1 *}, \beta_{r}^{2 *}, \lambda_{j}^{1 *}\right.$, $\left.\lambda_{j}^{2 *}, \mu_{j}^{1 *}, \mu_{j}^{2 *}\right)$ is an optimal solution for Model (5), then $\left(\theta_{i}^{*}=\frac{\theta_{i}^{1 *}}{t_{1}}=\frac{\theta_{i}^{2 *}}{t_{2}}, \varphi_{d}^{*}=\frac{\varphi_{d}^{1 *}}{t_{1}}=\frac{\varphi_{d}^{2 *}}{t_{2}}, \bar{\varphi}_{d}^{*}=\frac{\bar{\varphi}_{d}^{1 *}}{t_{1}}=\frac{\bar{\varphi}_{d}^{2 *}}{t_{2}}, \quad \beta_{r}^{*}=\right.$ $\left.\frac{\beta_{r}^{1 *}}{t_{1}}=\frac{\beta_{r}^{2 *}}{t_{2}}, \lambda_{j}^{*}=\frac{\lambda_{j}^{1 *}}{t_{1}}=\frac{\lambda_{j}^{2 *}}{t_{2}}, \mu_{j}^{*}=\frac{\mu_{j}^{1 *}}{t_{1}}=\frac{\mu_{j}^{2 *}}{t_{2}}\right)$ is an optimal solution for Model (4) when $\hat{w}_{1}=\hat{w}_{2}=\frac{1}{2}$.

Proof Suppose that $\left(\theta_{i}^{1 *}, \theta_{i}^{2 *}, \varphi_{d}^{1 *}, \varphi_{d}^{2 *}, \bar{\varphi}_{d}^{1 *}, \bar{\varphi}_{d}^{2 *}, \beta_{r}^{1 *}, \beta_{r}^{2 *}\right.$, $\left.\lambda_{j}^{1 *}, \lambda_{j}^{2 *}, \mu_{j}^{1 *}, \mu_{j}^{2 *}\right)$ is an optimal solution for Model (5). Obviously, $\left(\theta_{i}^{*}, \varphi_{d}^{*}, \bar{\varphi}_{d}^{*}, \beta_{r}^{*}, \lambda_{j}^{*}, \mu_{j}^{*}\right)$ is a feasible solution for Model (4) when $\hat{w}_{1}=\hat{w}_{2}=\frac{1}{2}$. For the selected optimal solution of Model (5):

$$
\begin{aligned}
& \exists \lambda_{j}^{1 *}, \lambda_{j}^{2 *} \geq 0,1 \lambda_{j}^{1 *}=t_{1}, 1 \lambda_{j}^{2 *}=t_{2} \text { and } \exists \mu_{j}^{1 *}, \mu_{j}^{2 *} \geq 0,1 \mu_{j}^{1 *} \\
& =t_{1}, 1 \mu_{j}^{2 *}=t_{2}, \\
& \text { s.t. } \quad \theta_{i}^{1 *} \leq t_{1}, \theta_{i}^{2 *} \leq t_{2}, \varphi_{d}^{1 *} \geq t_{1}, \varphi_{d}^{2 *} \geq t_{2}, \bar{\varphi}_{d}^{1 *} \leq t_{1}, \bar{\varphi}_{d}^{2 *} \\
& \leq t_{2}, \beta_{r}^{1 *} \geq t_{1}, \beta_{r}^{2 *} \geq t_{2} \\
& \frac{1}{2}\left(\frac{1}{m} \sum_{i=1}^{m} \theta_{i}^{1 *}+\frac{1}{D} \sum_{d=1}^{D} \bar{\varphi}_{d}^{2 *}\right) \\
& \leq \frac{1}{2}\left(\frac{1}{m} \sum_{i=1}^{m} \theta_{i}^{1}+\frac{1}{D} \sum_{d=1}^{D} \bar{\varphi}_{d}^{2}\right) .
\end{aligned}
$$

Additionally,

$$
\begin{aligned}
t_{1} & =\frac{1}{\frac{1}{D} \sum_{d=1}^{D} \varphi_{d}} \geq 0, t_{2}=\frac{1}{\frac{1}{s} \sum_{r=1}^{s} \beta_{r}} \geq 0, \theta_{i}^{*}=\frac{\theta_{i}^{1 *}}{t_{1}} \text { and } \bar{\varphi}_{d}^{*} \\
& =\frac{\bar{\varphi}_{d}^{2 *}}{t_{2}} .
\end{aligned}
$$

It is noted that

$$
\begin{aligned}
& \frac{1}{2}\left(\frac{1}{m} \sum_{i=1}^{m} t_{1} \theta_{i}^{*}+\frac{1}{D} \sum_{d=1}^{D} t_{2} \bar{\varphi}_{d}^{*}\right) \\
& \leq \frac{1}{2}\left(\frac{1}{m} \sum_{i=1}^{m} t_{1} \theta_{i}+\frac{1}{D} \sum_{d=1}^{D} t_{2} \bar{\varphi}_{d}\right) \\
& \Rightarrow \frac{1}{2}\left(\frac{\frac{1}{m} \sum_{i=1}^{m} \theta_{i}^{*}}{\frac{1}{D} \sum_{d=1}^{D} \varphi_{d}^{*}}+\frac{\frac{1}{D} \sum_{d=1}^{D} \bar{\varphi}_{d}^{*}}{\frac{1}{s} \sum_{r=1}^{s} \beta_{r}^{*}}\right) \\
& \leq \frac{1}{2}\left(\frac{\frac{1}{m} \sum_{i=1}^{m} \theta_{i}}{\frac{1}{D} \sum_{d=1}^{D} \varphi_{d}}+\frac{\frac{1}{D} \sum_{d=1}^{D} \bar{\varphi}_{d}}{\frac{1}{s} \sum_{r=1}^{s} \beta_{r}}\right) .
\end{aligned}
$$

Let $\hat{w}_{1}=\hat{w}_{2}=\frac{1}{2}$; thus

$$
\begin{aligned}
& \left(\hat{w}_{1} \frac{\frac{1}{m} \sum_{i=1}^{m} \theta_{i}^{*}}{\frac{1}{D} \sum_{d=1}^{D} \varphi_{d}^{*}}+\hat{w}_{2} \frac{\frac{1}{D} \sum_{d=1}^{D} \bar{\varphi}_{d}^{*}}{\frac{1}{s} \sum_{r=1}^{s} \beta_{r}^{*}}\right) \\
& \leq\left(\hat{w}_{1} \frac{\frac{1}{m} \sum_{i=1}^{m} \theta_{i}}{\frac{1}{D} \sum_{d=1}^{D} \varphi_{d}}+\hat{w}_{2} \frac{\frac{1}{D} \sum_{d=1}^{D} \bar{\varphi}_{d}}{\frac{1}{s} \sum_{r=1}^{s} \beta_{r}}\right) .
\end{aligned}
$$

Therefore $\left(\theta_{i}^{*}, \varphi_{d}^{*}, \bar{\varphi}_{d}^{*}, \beta_{r}^{*}, \lambda_{j}^{*}, \mu_{j}^{*}\right)$ is an optimal solution for Model (4). Proof is complete. 


\section{References}

[1] Charnes A, Cooper W W and Rhodes E 1978 Measuring the efficiency of decision making units. European Journal of Operational Research 2(6): 429-444

[2] Cook W D and Seiford L M 2009 Data envelopment analysis (DEA) - thirty years on. European Journal of Operational Research 192(1): 1-17

[3] García-Sánchez I M 2007 Efficiency and effectiveness of Spanish football teams: a three-stage DEA approach. Central European Journal of Operations Research. 15(1): 21-45

[4] Çelen A 2013 Efficiency and productivity (TFP) of the Turkish electricity distribution companies: an application of two-stage (DEA \& Tobit) analysis. Energy Policy 63: 300-310

[5] Wanke P F 2013 Physical infrastructure and shipment consolidation efficiency drivers in Brazilian ports: a two-stage network-DEA approach. Transport Policy 29: 145-153

[6] Wanke P and Barros C 2014 Two-stage DEA: an application to major Brazilian banks. Expert Systems with Applications 41(5): 2337-2344

[7] Ebrahimnejad A, Tavana M, Lotfi F H, Shahverdi R and Yousefpour M 2014 A three-stage data envelopment analysis model with application to banking industry. Measurement 49: 308-319

[8] Mozaffari M R, Gerami J and Jablonsky J 2014 Relationship between DEA models without explicit inputs and DEA-R models. Central European Journal of Operations Research 22(1): 1-12

[9] Hatami-Marbini A, Ebrahimnejad A and Lozano S 2017 Fuzzy efficiency measures in data envelopment analysis using lexicographic multi-objective approach. Computers \& Industrial Engineering 105: 362-376

[10] Tavana M, Ebrahimnejad A, Santos-Arteaga F J, Mansourzadeh S M and Matin R K 2016 A hybrid DEA-MOLP model for public school assessment and closure decision in the City of Philadelphia. Socio-Economic Planning Sciences 61: 70-89

[11] Ozcan Y A and Khushalani J 2017 Assessing efficiency of public health and medical care provision in OECD countries after a decade of reform. Central European Journal of Operations Research 25(2): 325-343

[12] Gutiérrez E, Aguilera E, Lozano S and Guzmán G I 2017 A two-stage DEA approach for quantifying and analysing the inefficiency of conventional and organic rain-fed cereals in Spain. Journal of Cleaner Production 149: 335-348

[13] Ozer S and Zorlu H 2011 Identification of bilinear systems using differential evolution algorithm. Sadhana 36(3): 281

[14] Charnes A, Cooper W W, Golany B, Halek R., Klopp G, Schmitz E and Thomas D 1986 Two phase data envelopment analysis approaches to policy evaluation and management of army recruiting activities: trade-offs between joint services and army advertising. Center for Cybernetic Studies. University of Texas-Austin Austin, Texas, USA

[15] Lovell C K, Walters L C and Wood L L 1994 Stratified models of education production using modified DEA and regression analysis. In: Data envelopment analysis: theory, methodology, and applications. Dordrecht: Springer, pp. 329-351

[16] Seiford L M and Zhu J 1999 Profitability and marketability of the top 55 US commercial banks. Management Science 45(9): 1270-1288
[17] Sexton T R and Lewis H F 2003 Two-stage DEA: an application to major league baseball. Journal of Productivity Analysis 19(2-3): 227-249

[18] Wang C H, Gopal R D and Zionts S 1997 Use of data envelopment analysis in assessing information technology impact on firm performance. Annals of Operations Research 73: 191-213

[19] Rho S and An J 2007 Evaluating the efficiency of a two-stage production process using data envelopment analysis. International Transactions in Operational Research 14(5): 395-410

[20] Kao C and Hwang S N 2008 Efficiency decomposition in two-stage data envelopment analysis: an application to nonlife insurance companies in Taiwan. European Journal of Operational Research 185(1): 418-429

[21] Liang L, Cook W D and Zhu J 2008 DEA models for twostage processes: game approach and efficiency decomposition. Naval Research Logistics 55(7): 643-653

[22] Tone K and Tsutsui M 2009 Network DEA: a slacks-based measure approach. European Journal of Operational Research 197(1): 243-252

[23] Chen Y, Cook W D and Zhu J 2010 Deriving the DEA frontier for two-stage processes. European Journal of Operational Research 202(1): 138-142

[24] Chen Y, Cook W D, Li N and Zhu J 2009 Additive efficiency decomposition in two-stage DEA. European Journal of Operational Research 196(3): 1170-1176

[25] Chen Y, Liang L and Zhu J 2009 Equivalence in two-stage DEA approaches. European Journal of Operational Research. 193(2): 600-604

[26] Chen Y and Zhu J 2004 Measuring information technology's indirect impact on firm performance. Information Technology and Management 5(1-2): 9-22

[27] Wang Y M and Chin K S 2010 Some alternative DEA models for two-stage process. Expert Systems with Applications 37(12): 8799-8808

[28] Liu S T 2011 A note on efficiency decomposition in twostage data envelopment analysis. European Journal of Operational Research 212(3): 606-608

[29] Du J, Liang L, Chen Y, Cook W D and Zhu J 2011 A bargaining game model for measuring performance of twostage network structures. European Journal of Operational Research 210(2): 390-397

[30] Yu Y, Shi Q F and Song J 2013 A note on some alternative DEA models for two-stage process. Expert Systems with Applications 40(10): 4268-4269

[31] Lewis H F, Mallikarjun S and Sexton T R 2013 Unoriented two-stage DEA: the case of the oscillating intermediate products. European Journal of Operational Research 229(2): 529-539

[32] Yu Y and Shi Q 2014 Two-stage DEA model with additional input in the second stage and part of intermediate products as final output. Expert Systems with Applications 41(15): 6570-6574

[33] Ahmadzadeh R, Kordrostami S and Amirteimoori A 2017 Evaluating the efficiency of a two-stage network structure with the use of fractional programming. Discrete Mathematics, Algorithms and Applications 9(03): 1750034

[34] Maghbouli M, Amirteimoori A and Kordrostami S 2014 Two-stage network structures with undesirable outputs: a DEA based approach Measurement 48: 109-118 
[35] Liu W, Zhou Z, Ma C, Liu D and Shen W 2015 Two-stage DEA models with undesirable input-intermediate-outputs. Omega 56: 74-87

[36] Jianfeng M A 2015 A two-stage DEA model considering shared inputs and free intermediate measures. Expert Systems with Applications 42(9): 4339-4347

[37] Lim S and Zhu J 2016 A note on two-stage network DEA model: frontier projection and duality. European Journal of Operational Research 248(1): 342-346

[38] Mahdiloo M, Jafarzadeh A H, Saen R F, Tatham P and Fisher R 2016 A multiple criteria approach to two-stage data envelopment analysis. Transportation Research Part D: Transport and Environment 46: 317-327

[39] Guo C, Shureshjani R A, Foroughi A A and Zhu J 2017 Decomposition weights and overall efficiency in two-stage additive network DEA. European Journal of Operational Research 257(3): 896-906

[40] Wanke P F, Hadi-Vencheh A and Forghani A 2017 A DDF based model for efficiency evaluation in two-stage DEA. Optimization Letters 12(5): 1029-1044

[41] Chen Y, Cook W D, Kao C and Zhu J 2013 Network DEA pitfall: divisional efficiency and frontier projection under general network structures. European Journal of Operational Research 226: 507-515

[42] Amirteimoori A 2013 A DEA two-stage decision processes with shared resources. Central European Journal of Operations Research 21(1): 141-151

[43] Soltanifar M, Jahanshahloo G R, Lotfi F H and Mansourzadeh S M 2013 On efficiency in convex hull of DMUs. Applied Mathematical Modelling 37(4): 2267-2278 